\title{
On Limiting Procedures
}

\author{
M.M. YANASE \\ Saphia University
}

\section{$\langle 1\rangle$ Introduction}

In recent article on continuous superselection rule and the measuring process in Quantum mechanics ${ }^{(1)}, \mathrm{H}$. Araki showed very clearly the necessity of introducing a new mathematical formalism to describe a macroscopic system in order to explain the measuring process consistently. He named it continuous superselection rule, because it is a natural extension of well-known superselection rule, from its discrete to costinuous case. The problems on this formalism and its application in general were already discussed in earlier publications ${ }^{(2)(6)}$.

In this article we want to discuss on the problems of limiting procedures, which are used in the description of Araki(1), from more philosophical point of view. This problem is closely related to the problems on change of scales from microscopic to macroscopic order. The problem on changing from microscopic to cosmological scales will also be discussed.

In section 2, we take the problem of taking limit to infinity of variables like time, momenta or mass.

In section 3 , the problem of changing scales in space and time will be discussed from more general point of view.

The problem of so called "Inflation" in the discussion on big-bang theory will be touched on.

\section{$\langle 2\rangle$ Limiting procedures in time, momenta and mass}

H. Araki, in the above mentioned articles ${ }^{(1)}$, uses Rieman-Lebesgue Lemma to prove the transition of the separating apparatus from a pure state to a mixture in Stern-Gerlach type measurement. The essense of the Riemam-Lebesgne Lemma is the vanishing of interference term, if time variable $t$ goes to infinity;

$$
\lim _{t \rightarrow \infty} \int_{-\infty}^{+\infty} f(\lambda) e^{i \lambda t} d \lambda=0
$$

He stresses, from mathematical reason, that $t \rightarrow \infty$ is essential for the proof.

The same argument is applied to the case of Machida-Namiki model. In this

Annals of the Japan Association for Philosophy of Science, March 1988

$$
-125-
$$


case, not only $t$ goes to infinity, but also the momenta of two colliding particles concerned, as well as the difference of the two momenta, and the mass of one (target) particle go to infinity.

All these quantities are, physically speaking, large but finite. However, mathematically speaking, they are considered as infinities. H. Araki himself admits that, because of these estimations of quantities concerned, "the reduction of wave packets is approximate", and it "depends on the smoothness of various function as well as actual order of magnitudes for these large quantities".

First we consider limiting procedures in time. Physics in general, but particle physics in particular, limiting procedures are often used as a legitimate mathematical argument, even if, physically speaking, quantities concerned are large, but not infinite.

Take for example, S-matrix theory. In-state and Out-state before and after the collision are $-\infty$ and $+\infty$ in time respectively. We consider this procedure of taking limit in both directions of time legitimate, because the difference between mathematical infiniteness and physical finiteness does not change the result of the argument. Infinities which appear in re-normalization theories have quite different characters. They should be not only finite but comparable order of magnitude with other quantities.

Already in classical mechanics, the infinity appears in time variable. In damping oscillation, for example, if the friction or resistance is proportional to velocity of the particle, the oscillating mass particle, mathematically speaking, never stops within finite time. In this case, we do not care too much on discrepancy between mathematical and physical situation. A damping particle either will never stop, but the amplitude will become negligibly small, or it will stop because the friction or resistance will not be exactly proportional to the velocity of the particle ${ }^{(3)}$.

A possible objection may come from the argument by time reversal. Let us take the case, where the resistance is exactly proportional to velocity. If we operate time reversal to the system concerned, the particle will increase its oscillating amplitude and trace back the past trajectory, however small the amplitude at the moment of the time reversal might be, whereas if the particle stands still then, it will never start to move again, because it "forgot" the past experience.

In S-matrix theory, the objection does not work, because of the time symmetry of the whole theory.

The objection against the argument of Araki concerning time reversal might be raised in the following way. Like in a damping oscillator, the interference term will never become zero, so that if we operate time reversal to the system, i.e. let the particle go through the magnetic field of the same direction and magnitude again, a mixture will become again a pure state. In other words, reduction of wave packet will still occur, when the system is observed after time reversal, because its state is 
not exactly a mixture.

This objection was actually made by Jauch, Wigner and Yanase against another argument of measurement process by Daneri, Loinger and Prosperi ${ }^{(4)}$.

The difference between the argument of Daneri, Loinger and Prosperi and that of Araki is in the expression of the external magnetic field. According to Araki, the external magenetic field can not be controlled with absolute accuracy ${ }^{(1)}$. This is the reason why it is expressed by way of continuous superselection rule, whereas in the former argument it is not the case. Therefore, this objection is not valid to the Araki's argument.

Next, we want to discuss on infinities which appear to mass or momenta in Araki's arguments.

The mathematical procedures are the same as in the case of time. The difference in this case is the physical meaning. These quantities are not dynamical variables like time variable $t$, so that the limiting processes are not physical, but only mathematical. This distinction appears in classical "dilemma" of Achiles and Tortoise. The limiting procedure in this case is mathematical division of a finite time into an infinite series, but it is not a physical process.

Therefore in these cases time-reversal-like objection will not occur. The only physical problem is, as Araki mentions, that of orders of magnitudes for these large quantities $^{(1)}$.

\section{〈3〉 Change of scales of physical quantities from microscopic to macroscopic order}

One essential element of Araki's formalism is, as mentioned above, the introduction of continuous superselection rule for a macroscopic system. We discussed on this before from a general point of view ${ }^{(2)}$.

It is clear that we can describe a macroscopic system by a ray in a Hilbert space of continous superselection rule, i.e. a Hilbert space which is a direct intergral of uncountable many Hilbert spaces $H_{\lambda}$, i.e.

$$
H=\int^{\oplus} H_{\lambda} d \mu(\lambda)
$$

It is also shown that classical mechanics which is valid for macroscopic systems can be described by using centre of operators-which are commutative-in "large" Hilbert space $H_{\lambda}{ }^{(2)}$.

The problem is how we can get the norm or criteria for the change of scales by which we can go over from microscopic to macroscopic description. In other words, we need a norm for a change from one Hilbert space formalism to continous superselection rule. 
One natural solution of this problem is the use of universal constants like Planck's constant $h$, or a mass of an electron. Like in the case of Araki's model of coliding particles, if mass of the particle concerned is large campared with mass of an electon, we can apply continuous superselection rule on this system. Therefore, even if the system is composed of a single particle, we can consider it as a macroscopic system, if mass is large enough in the sense just mentioned.

Actually in this model, physically speaking, the system is composed of great many number of particles, but we neglect all the interactions among themselves, because they are not essential for the discussion, and consider the whole system as a single heavy particle in the centre of mass. This is actually the meaning of a rigid body in classical mechanics.

Secondly, as already pointed out before ${ }^{(2)}$, the fuzzy concept should be applied to this system, because the number of particles and the size of this system are not absolutely fixed. Therefore, we introduce continuous superselection rule to this system, and consider concepts like external magnetic field as fuzzy ones.

A norm for the change of scale concerning time and space will be the comparison of the orders of magnitude for the model and those of elementary particles. For time, it will be order of $10^{-20} \mathrm{sec}$., for space, order of $10^{-13} \mathrm{~cm}$. Therefore, the order of magnitude for the change in time will be $10^{20}$, in space it will be $10^{13}$. We consider that it is legitimate to make these orders of magnitude will be infinities in mathematical sense.

Finally, change of scale to cosmological order is becomming an interesting problem. According to "inflation" theory, at the time of Big-Bang, the scale of cosmological order in space will increase at least by $10^{31}$, in a very small order of time of $10^{-40(5)}$.

Still we can not simply apply the criteria of the change of order from microscopic to a macroscopic scale, because in this case even though the order of change of scale is much larger, it should be applied for the same system in consideration.

Therefore, we need a spacial discussion on this case, both for the order of change of scale in space and time, and the applicability of continuous superselection rule. On these points we should investigate further.

The most part of this article is written during my stay at the Vatican Observatory in Castel Gandolfo. The auther is very grateful to Rev. George Coyne, S.J., the Diretor, and the staff of the Observatory for their kind hospitality and help.

\section{References}

(1) H. Araki : "Fundamental Aspects of Quantum Theory", V. Gorini \& A. Frigerio, Eds., p. 23 (1896).

(2) M.M. Yanase: Ann. Jap. Assoc. Phil. Sci. Vol. 6, pp. 83-89 (1982). 
S. Machida \& M. Namiki : Prog. Theoret. Phys., Vol. 63, pp. 1457-1473 (1980).

S. Machida \& M. Namiki : Proc. of ISQM-Tokyo '86, M. Namiki et al., Eds., (1987).

(3) cf. Any text book of Classical mechanics.

(4) J.M. Jauch, E.P. Wigner and M.M. Yanase: Nuovo Cimento, Vol. 48B pp. 114-151 (1967).

(5) e.g. A.D. Linde : Particle Physics and Cosmology (given at MESON 50 : Kyoto International Symposium The Jubilee of the Meson Theory Kyoto, August 15-17, 1985).

(6) cf. also, M. Namiki : Many-Hilbert-Spaces Theory of Quantum Measurements, (1987) Preprint.

M. Ozawa : Ann. Jap. Assoc. Phil. Sci., Vol. 18 No.1 pp. 35-43 (1987), (in Japanese). 